\title{
Uncertainty estimation in equality-constrained MAP and maximum likelihood estimation with applications to system identification and state estimation
}

\author{
Dimas Abreu Archanjo Dutra \\ Departamento de Engenharia Mecânica - Universidade Federal de Minas Gerais — Belo Horizonte, MG, Brasil
}

\begin{abstract}
In unconstrained maximum a posteriori (MAP) and maximum likelihood estimation, the inverse of minus the merit-function Hessian matrix is an approximation of the estimate covariance matrix. In the Bayesian context of MAP estimation, it is the covariance of a normal approximation of the posterior around the mode; while in maximum likelihood estimation, it an approximation of the inverse Fisher information matrix, to which the covariance of efficient estimators converge. These measures are routinely used in system identification to evaluate the estimate uncertainties and diagnose problems such as overparametrization, improper excitation and unidentifiability. A wide variety of estimation problems in systems and control, however, can be formulated as equality-constrained optimizations with additional decision variables to exploit parallelism in computer hardware, simplify implementation and increase the convergence basin and efficiency of the nonlinear program solver. The introduction of the extra variables, however, dissociates the inverse Hessian from the covariance matrix. Instead, submatrices of the inverse Hessian of the constrained-problem's Lagrangian must be used. In this paper, we derive these relationships, showing how the estimates' covariance can be estimated directly from the augmented problem. Application examples are shown in system identification with the output-error method and joint state-path and parameter estimation.
\end{abstract}

Key words: System model validation; measures of model fit; estimation theory; statistical analysis; system identification.

\section{Introduction}

When formulating a nonlinear optimization problem, function composition in the merit function can be replaced by the introduction of additional decision variables and equality constraints. This general technique can simplify the implementation, transform a dense optimization problem into a sparse one, and help overcome local optima and convergence issues [Dutra, 2019, Ribeiro et al., 2019]. In the output-error method for system identification, this principle underlies the use of collocation and multiple shooting in continuous-time [Bock, 1980, 1983; Betts and Huffman, 2003; Williams and Trivailo, 2005; Betts, 2010, Chap. 5; Boisvert et al., 2012; Dutra, 2019] and discrete-time systems [Ribeiro and Aguirre, 2017]. The approach is pretty general, however, and has been applied to other state [López-Negrete and Biegler, 2012, Dutra et al., 2014] and joint state and parameter estimators [Dutra et al., 2012, 2017].

Email address: dimasad@ufmg.br (Dimas Abreu Archanjo Dutra).
In unconstrained maximum a posteriori (MAP) and maximum likelihood (ML) estimation, the inverse of the merit-function Hessian matrix is an approximation of the estimates' covariance matrix. This is used extensively to obtain estimate correlations, uncertainties, and significance in system identification [Ljung, 1999, Chap. 16] and its applications to aircraft [Maine and Iliff, 1981; Klein and Morelli, 2006, Sec. 6.3.3; Jategaonkar, 2015, Sec. 11.2]. The covariance matrix, meritfunction Hessian, and measures derived from them can, in turn, be used to diagnose overparametrization, unidentifiability, improper excitation, and inadequate model postulates [Mehra, 1974, Söderström, 1975, Stoica and Söderström, 1982]. From the standpoint of the optimization problem, the Hessian quantifies how deep or shallow the basin around the optimum is. A small eigenvalue means that a certain parameter or parameter combination changes the posterior density or the likelihood very little and, consequently, is not well estimated from the test data.

There are also formal interpretations to this intuitive concept that justify the use of the Hessian matrix to 
quantify the estimates' uncertainty. In classical statistics, the parameters are considered unknown deterministic quantities and their data-dependent estimates are random variables. Under some regularity conditions, maximum likelihood estimators are asymptotically consistent, unbiased, efficient, and normally distributed [see, e.g., Cramér, 1946, Sec. 33.3; Wald, 1949; Wilks, 1962, Sec. 12.3]. The regularity conditions are associated with a well-posed ML estimator for sufficient data, such as having unique global maxima for all large enough datasets. The covariance of efficient estimators is given by the inverse of the Fisher information matrix. If the estimator is consistent, minus the Hessian of the log-likelihood at the optima converges to the Fisher information by the law of large numbers.

These conditions apply for a wide class of prediction error methods in system identification [Kashyap, 1970, Caines and Ljung, 1976, Ljung, 1999, Chaps. 8-9] and are assumed to hold for aircraft system identification methods such as the output error method and the filter error method [Murphy, 1985, Sec. III.B-2; Jategaonkar, 2015, Appx. D]. This makes the merit-function Hessian an asymptotic measure of the estimate uncertainties for a wide range of problems in systems and control. Its use in the practice of aircraft system identification is supported by experimental evidence and simulated analyses [see, e.g., Maine and Iliff, 1981].

Under a Bayesian framework of MAP estimation, the merit-function is the log-posterior, the logarithm of the posterior probability density. Under some regularity conditions, the Bayesian Central Limit Theorem [Ando, 2010, Sec. 3.2.1], also known as the Bernstein-von Mises Theorem [van der Vaart, 1998, Sec. 10.2] states that the posterior converges asymptotically to a normal distribution centered around the mode, with covariance given by minus the inverse merit-function Hessian. This is a Bayesian analog of the limiting behavior of the ML estimator which, as data grows, also converges to the same value as the posterior mode.

The introduction of aditional variables and equality constraints in approaches like collocation and multiple shooting, however, dissociates the merit-function Hessian from the Fisher information matrix. In this work, we prove that the inverse Hessian of the equivalent unaugmented problem is equal to a submatrix of the inverse Hessian of the Lagrangian of the augmented, equality-constrained, problem. We also show how to approximate the dependent variables' covariance, based on a linear approximation of the constraints, from the Lagrangian Hessian. This allows the computation of the estimate uncertainties directly from the functions and results used to solve the augmented nonlinear program (NLP). We note that, although our motivation is system identification and state estimation, the results are general and apply to any equality-constrained maximum likelihood or maximum a posteriori estimator, even beyond the field of systems and automatic control.

A computationally similar, although conceptually different solution to the same problem is given by Ramsay et al. [2007, Sec. 2.6], Pirnay et al. [2012, Sec. 4] and López-Negrete and Biegler [2012, Sec. 2.3]. As explained in detail by Bard [1974, Sec. 7.7], it consists of evaluating the first-order change in the estimates generated by a stochastic perturbation to the data. The covariance of the estimate variation then gives, approximatelly, the estimate uncertainties, especially when that variation is small and hence the linear approximation is reasonable. In nonlinear least-square problems both approaches give the same result, although in non-Gaussian ML problems like those associated with robust estimation they may differ. It should be noted, however, that this approach of considering stochastic perturbations to the data does not rely on the assumptions of consistency and efficiency of the estimator, so it is applicable to other optimizationbased methods besides MAP and maximum likelihood [Bard, 1974, Sec. 7.5].

The remainder of this article is organized as follows. In Sec. 2 we define the reduced and augmented estimation problems and present the theoretical result. In Sec. 3 we present applications of the result to the output-error method of system identification, joint MAP state-path and parameter estimation in stochastic differential equations (SDEs), and Markov chain Monte Carlo (MCMC) sampling of state-paths and parameters in SDEs. The paper concludes with the final remarks in Sec. 4 .

\section{Theoretical result}

\subsection{Notation}

For a scalar function $f: \mathbb{R}^{n} \times \mathbb{R}^{m} \rightarrow \mathbb{R}$ of two vectorvalued arguments, $f(x, y)$, we denote its gradient with respect to $x$ by $\nabla_{x} f(a, b) \in \mathbb{R}^{n}$ and Hessian matrix with respect to $x$ and $y$ by $\nabla_{x y}^{2} f(a, b) \in \mathbb{R}^{n \times m}$ :

$$
\begin{gathered}
{\left[\nabla_{x} f(a, b)\right]_{i}=\left.\frac{\partial f}{\partial x_{i}}\right|_{\substack{x=a \\
y=b}},} \\
{\left[\nabla_{x y}^{2} f(a, b)\right]_{i j}=\left.\frac{\partial^{2} f}{\partial x_{i} \partial y_{j}}\right|_{\substack{x=a \\
y=b}} .}
\end{gathered} .
$$

Similarly, for vector-valued functions $g: \mathbb{R}^{n} \times \mathbb{R}^{m} \rightarrow \mathbb{R}^{p}$ of two vector-valued variables $g(x, y)$, we define the Jacobian matrix with respect to $x$ as $\nabla_{x} g(a, b) \in \mathbb{R}^{p \times n}$ :

$$
\left[\nabla_{x} g(a, b)\right]_{i j}=\left.\frac{\partial g_{i}}{\partial x_{j}}\right|_{\substack{x=a \\ y=b}}
$$

For functions of only one argument the subscript to the nabla symbol may be omitted. In addition, $\nabla_{x x}^{2}$ will be shortened to $\nabla_{x}^{2}$. 


\subsection{Augmented and reduced problem definitions}

We now begin with the definition of the estimation problems under consideration. Our starting point is the augmented problem, written in terms of the independent variables $p \in \mathbb{P} \subseteq \mathbb{R}^{n}$ and the dependent variables $q \in \mathbb{Q} \subseteq \mathbb{R}^{m}$ :

$$
\begin{array}{cl}
\underset{p \in \mathbb{P}, q \in \mathbb{Q}}{\operatorname{maximize}} & \ell(p, q) \\
\text { subject to } & g(p, q)=0,
\end{array}
$$

where $\ell: \mathbb{P} \times \mathbb{Q} \rightarrow \mathbb{R}$ is the merit function and the equality constraints are encoded by $g: \mathbb{P} \times \mathbb{Q} \rightarrow \mathbb{R}^{m}$. To simplify notation, we let $z \in \mathbb{Z}:=\mathbb{P} \times \mathbb{Q}$ denote the full decision variable vector of the augmented problem, i.e., $z=\left[p^{\top} q^{\top}\right]^{\top}$ and let $\ell$ and $g$ be written in terms of either $z$ or the $(p, q)$ pair.

We consider only well-posed problems which can be rewritten as unconstrained optimizations only in terms of the independent variables $p$, at least around the optimum. Formally, this translates to the assumptions below.

\section{Assumption 1.}

a. There exists a unique solution $p^{*}, q^{*}$ to the problem defined in Eq. (1), and it lies in the interior of the search space, $p^{*} \in \mathbb{P}^{\circ}, q^{*} \in \mathbb{Q}^{\circ}$.

$b$. The merit $\ell$ and constraint $g$ are twice continuously differentiable (of class $C^{2}$ ) with respect to all arguments at a neighbourhood $\mathbb{P}^{*} \times \mathbb{Q}^{*} \subseteq \mathbb{P} \times \mathbb{Q}$ of the optimum $z^{*}=\left[p^{* \top} q^{* \top}\right]^{\top}$.

c. The Jacobian matrix $\nabla_{q} g\left(z^{*}\right)$ of $g$ with respect to the dependent variables $q$, evaluated at the optimum, is invertible.

Assum. 1 implies that the implicit function theorem can be used, consequently there exists a unique explicit solution to the constraints around the optimum.

Corollary 2 (of the implicit and inverse function theorems, Krantz and Parks, 2003, Thms. 3.3.1 and 3.3.2). If Assum. 1 holds then, for a neighbourhood $\mathbb{P}^{*} \subseteq \mathbb{P}$ of $p^{*}$, there exists a unique twice continuously differentiable (of class $C^{2}$ ) function $w: \mathbb{P}^{*} \rightarrow \mathbb{R}^{n+m}$ such that $w\left(p^{*}\right)=z^{*}$ and

$$
g(w(p))=0 \quad \forall p \in \mathbb{P}^{*} .
$$

Furthermore, the Jacobian matrix of $w$ at the optimum is

$$
\nabla w\left(p^{*}\right)=\left[\begin{array}{c}
\mathrm{I}_{n} \\
-\nabla_{q} g^{-1} \nabla_{p} g
\end{array}\right]_{z=z^{*}} .
$$

A consequence of Cor. 2 is that, at least in the neighborhood of the solution, the optimization problem can be rewritten in a reduced form without constraints, in terms of only the independent variables $p$ :

$$
\underset{p \in \mathbb{P}^{*}}{\operatorname{maximize}} \tilde{\ell}(p)
$$

where the reduced merit $\tilde{\ell}: \mathbb{P}^{*} \rightarrow \mathbb{R}$ is of class $C^{2}$ and given by

$$
\tilde{\ell}(p):=\ell(w(p)) \quad \forall p \in \mathbb{P}^{*} .
$$

Both problems are equivalent in terms of having the same solution.

\subsection{Definition of the Lagrangian and bordered Hessian}

We now define the Lagrangian $L: \mathbb{Z} \times \mathbb{R}^{m} \rightarrow \mathbb{R}$ of the augmented equality-constrained problem (1) so that the theorem can be stated:

$$
L(z, \lambda):=\ell(z)+g(z)^{\top} \lambda
$$

where $\lambda \in \mathbb{R}^{m}$ are the Lagrange multipliers. A standard result of constrained optimization that follows from Assum. 1 is that a necessary condition for optimality is the existence of $\lambda^{*}$ such that

$$
\nabla_{z} L\left(z^{*}, \lambda^{*}\right)=\nabla \ell\left(z^{*}\right)+\nabla g\left(z^{*}\right)^{\top} \lambda^{*}=0 .
$$

The Hessian matrix $H_{L}$ of the Lagrangian with respect to $z$ and $\lambda$ at the optimum is then given by

$$
H_{L}:=\left[\begin{array}{cc}
\nabla^{2} \ell+\sum_{i=1}^{m} \lambda_{i}^{*} \nabla^{2} g_{i} & \nabla g^{\top} \\
\nabla g & 0
\end{array}\right]_{z=z^{*}} .
$$

The $H_{L}$ matrix is sometimes called the bordered Hessian and is used in the numerical solution of the optimization problem. It is usually readily available to the user for evaluation after successful termination of the optimization.

\subsection{Equivalence of inverse Hessians}

We are now ready to present the main result of this article.

Theorem 3. If Assum. 1 holds, then both $\nabla^{2} \tilde{\ell}\left(p^{*}\right)$ and $H_{L}$ are invertible. Furthermore, the inverse of the Hessian matrix of the reduced problem (3) at the optimum $\left[\nabla^{2} \tilde{\ell}\left(p^{*}\right)\right]^{-1}$ is given by the first $n$ columns and $n$ rows of $H_{L}^{-1}$.

To prove Thm. 3 we obtain the expression of the inverse reduced Hessian and show that it is equal to a submatrix of the blockwise inversion of $H_{L}$. To obtain the reduced Hessian, we state a lemma often used to obtain sufficient 
second-order conditions for constrained optima. To simplify notation, in what follows all derivatives are evaluated at $p=p^{*}$ and $z=z^{*}$.

Lemma 4. The Hessian matrix of the reduced problem (3), at the optimum, is given by

$$
\nabla^{2} \tilde{\ell}=\nabla w^{\top}\left[\nabla^{2} \ell+\sum_{s=1}^{m} \lambda_{s}^{*} \nabla^{2} g_{s}\right] \nabla w
$$

Proof. Using the chain rule, we have that

$$
\begin{gathered}
\frac{\partial \tilde{\ell}}{\partial p_{i}}=\sum_{k=1}^{m+n} \frac{\partial \ell}{\partial z_{k}} \frac{\partial w_{k}}{\partial p_{i}} \\
\frac{\partial^{2} \tilde{\ell}}{\partial p_{i} \partial p_{j}}=\sum_{k=1}^{m+n} \sum_{r=1}^{m+n} \frac{\partial^{2} \ell}{\partial z_{k} \partial z_{r}} \frac{\partial w_{k}}{\partial p_{i}} \frac{\partial w_{r}}{\partial p_{j}}+\sum_{k=1}^{m+n} \frac{\partial \ell}{\partial z_{k}} \frac{\partial^{2} w_{k}}{\partial p_{i} \partial p_{j}} .
\end{gathered}
$$

Furthermore, from the first-order necessary conditions for the optimum (5) we have that

$$
\frac{\partial \ell}{\partial z_{k}}=-\sum_{s=1}^{m} \lambda_{s}^{*} \frac{\partial g_{s}}{\partial z_{k}}
$$

leading to the following expression for the last term of Eq. (8):

$$
\sum_{k=1}^{m+n} \frac{\partial \ell}{\partial z_{k}} \frac{\partial^{2} w_{k}}{\partial p_{i} \partial p_{j}}=-\sum_{s=1}^{m} \lambda_{s}^{*} \sum_{k=1}^{m+n} \frac{\partial g_{s}}{\partial z_{k}} \frac{\partial^{2} w_{k}}{\partial p_{i} \partial p_{j}}
$$

Next, define $\tilde{g}(p):=g(w(p))$. Applying the chain rule,

$$
\begin{gathered}
\frac{\partial \tilde{g}_{s}}{\partial p_{i}}=\sum_{k=1}^{n+m} \frac{\partial g_{s}}{\partial z_{k}} \frac{\partial w_{k}}{\partial p_{i}} \\
\frac{\partial^{2} \tilde{g}_{s}}{\partial p_{i} \partial p_{j}}=\sum_{k=1}^{n+m} \sum_{r=1}^{n+m} \frac{\partial^{2} g_{s}}{\partial z_{k} \partial z_{r}} \frac{\partial w_{k}}{\partial p_{i}} \frac{\partial w_{r}}{\partial p_{j}}+\sum_{k=1}^{n+m} \frac{\partial g_{s}}{\partial z_{k}} \frac{\partial^{2} w_{k}}{\partial p_{i} \partial p_{j}}
\end{gathered}
$$

As $w(p)$ is an explicit solution to the constraints, $\tilde{g}$ and all its derivatives are identically zero for all $p \in \mathbb{P}^{*}$, so

$$
\sum_{k=1}^{n+m} \frac{\partial g_{s}}{\partial z_{k}} \frac{\partial^{2} w_{k}}{\partial p_{i} \partial p_{j}}=-\sum_{k=1}^{n+m} \sum_{r=1}^{n+m} \frac{\partial^{2} g_{s}}{\partial z_{k} \partial z_{r}} \frac{\partial w_{k}}{\partial p_{i}} \frac{\partial w_{r}}{\partial p_{j}}
$$

Substituting (10) and (9) into (8), we obtain

$$
\begin{aligned}
& \frac{\partial^{2} \tilde{\ell}}{\partial p_{i} \partial p_{j}}= \\
& \quad \sum_{k=1}^{m+n} \sum_{r=1}^{m+n}\left(\frac{\partial^{2} \ell}{\partial z_{k} \partial z_{r}}+\sum_{s=1}^{m} \lambda_{s}^{*} \frac{\partial^{2} g_{s}}{\partial z_{k} \partial z_{r}}\right) \frac{\partial w_{k}}{\partial p_{i}} \frac{\partial w_{r}}{\partial p_{j}},
\end{aligned}
$$

which is the elementwise expression of (7).

Corollary 5. If Assum. 1 holds, then $\nabla^{2} \tilde{\ell}$ is invertible.

Proof. Note that $\nabla w$ is a basis for the null space of $\nabla g$, which can be verified by taking their product. Lemma 4 then amounts to the second-order sufficient and necessary conditions for constrained optimality [Nocedal and Wright, 2006, Thms. 12.5 and 12.6]. Additionally, as $z^{*}$ is assumed to be the unique constrained maximum, a consequence of the necessary conditions is that $\nabla^{2} \tilde{\ell}$ is negative-definite, hence invertible.

We are now ready to prove the main theorem.

Proof of Thm. 3. To begin, note that from the definition of $L$ in (4), we have that the bracketed expression in (7) is $\nabla_{z}^{2} L$, which has the following block structure:

$$
\nabla_{z}^{2} L=\nabla^{2} \ell+\sum_{i=1}^{m} \lambda_{i}^{*} \nabla^{2} g_{i}=\left[\begin{array}{cc}
\nabla_{p}^{2} L & \nabla_{q p}^{2} L^{\top} \\
\nabla_{q p}^{2} L & \nabla_{q}^{2} L
\end{array}\right]
$$

Substituting (2) and (12) into (7),

$$
\begin{aligned}
\nabla^{2} \tilde{\ell}=\nabla_{p}^{2} L- & \nabla_{q p}^{2} L^{\top} \nabla_{q}^{-1} \nabla_{p} g-\nabla_{p} g^{\top} \nabla_{q} g^{-\mathrm{\top}} \nabla_{q p}^{2} L \\
& +\nabla_{p} g^{\top} \nabla_{q} g^{-\mathrm{\top}} \nabla_{q}^{2} L \nabla_{q} g^{-1} \nabla_{p} g
\end{aligned}
$$

Next we obtain the expression of the first $n$ rows and $n$ columns of $H_{L}^{-1}$. The $H_{L}$ matrix of (6) has the following block structure:

$$
\begin{array}{rlrl}
H_{L} & =\left[\begin{array}{cc}
A & B \\
B^{\top} & D
\end{array}\right], & D & :=\left[\begin{array}{cc}
\nabla_{q}^{2} L & \nabla_{q} g^{\top} \\
\nabla_{q} g & 0
\end{array}\right], \\
A & :=\nabla_{p}^{2} L, & B:=\left[\nabla_{q p}^{2} L^{\top} \nabla_{p} g^{\top}\right] .
\end{array}
$$

From the blockwise inversion formula [Bernstein, 2009, Prop. 2.8.7], we have that if $D$ and $A-B D^{-1} B^{\top}$ are nonsingular, then so is $H_{L}$ and its inverse is given by (11). 


$$
H_{L}^{-1}=\left[\begin{array}{cc}
\left(A-B D^{-1} B^{\top}\right)^{-1} & -\left(A-B D^{-1} B^{\top}\right)^{-1} B D^{-1} \\
-D^{-1} B^{\top}\left(A-B D^{-1} B^{\top}\right)^{-1} & D^{-1}+D^{-1} B^{\top}\left(A-B D^{-1} B^{\top}\right)^{-1} B D^{-1}
\end{array}\right]
$$

As $\nabla_{q} g$ is invertible due to Assum. 1c, then $D$ is invertible,

$$
D^{-1}=\left[\begin{array}{cc}
0 & \nabla_{q} g^{-1} \\
\nabla_{q} g^{-\top} & -\nabla_{q} g^{-\mathrm{T}} \nabla_{q}^{2} L \nabla_{q} g^{-1}
\end{array}\right]
$$

which can be verified directly by taking the product. Again, taking the product and comparing to the righthand side of (13),

$$
A-B D^{-1} B^{\top}=\nabla^{2} \tilde{\ell}
$$

which by Cor. 5 is invertible. Consequently, the blockwise inversion formula is applicable and from (11) and (16) we have that $\left(\nabla^{2} \tilde{\ell}\right)^{-1}$ equals the first $n$ rows and $n$ columns of $H_{L}^{-1}$.

\subsection{Approximate covariance of all decision variables}

When the inverse Hessian is used as an approximation of the independent variables' covariance, a natural question that arises is if it can be used to obtain the covariance of the full vector of decision variables, or at least an estimate of. If a linear approximation of the constraints and, consequently, of the relationship between the independent and dependent variables $q$ and $p$ is used,

$$
w(p)-z^{*} \approx(\nabla w)\left(p-p^{*}\right)
$$

for $p$ in a neighbourhood of the optimum $p^{*}$. This approximation, together with the use of $\left(\nabla^{2} \tilde{\ell}\right)^{-1}$ for the covariance of the estimates, yields

$$
\begin{aligned}
\mathrm{E}\left[\left(z^{*}-\bar{z}\right)\left(z^{*}-\bar{z}\right)^{\top}\right] & \approx(\nabla w)\left(\nabla^{2} \tilde{\ell}\right)^{-1}(\nabla w)^{\top}, \\
\mathrm{E}\left[\left(z-z^{*}\right)\left(z-z^{*}\right)^{\top} \mid y\right] & \approx(\nabla w)\left(\nabla^{2} \tilde{\ell}\right)^{-1}(\nabla w)^{\top},
\end{aligned}
$$

in which $\bar{z}$ is the true value of the estimates, in the context of maximum likelihood estimation, and the expected value of $z^{*}$ if the estimator is unbiased. In a Bayesian context (17b), $z$ is the random variable being estimated, $y$ is the data, and the posterior mode $z^{*}$ is used as the mean.

The results of the previous section can be used to obtain an expression of the right-hand sides of (17) in terms of $H_{L}^{-1}$. These are summarized in the following Lemma.

Lemma 6. If Assum. 1 holds, then $(\nabla w)\left(\nabla^{2} \tilde{\ell}\right)^{-1}(\nabla w)^{\top}$ is given by the first $m+n$ rows and $n+m$ columns of $H_{L}^{-1}$.
Proof. Using the results obtained previously, a direct proof is possible. By substituting (14) and (15) into (11), we can obtain the expression of $H_{L}^{-1}$. Similarly, (2) and (13) can be substituted into $(\nabla w)\left(\nabla^{2} \tilde{\ell}\right)^{-1}(\nabla w)^{\top}$ and we can see that the statement holds.

It should be noted that the use of the approximation (17a) in the context of classical statistics and maximum likelihood estimation is better justified when the constraints are not data-dependent. In this case, the same function $w$ of Cor. 2 is valid for all realizations of the experiment. This occurs in the output-error method, for example, as the constraints encode the solution of a deterministic ordinary differential equation or difference equation.

An important issue in using these methods is that although in most large problems of practical interest the Lagrangian Hessian is sparse, its inverse is generally dense. A workaround to this, which we use in the example of Sec. 3.1, is to obtain individual columns of $H_{L}^{-1}$ by solving the linear system $H_{L} x=b$, in which $b$ is a column of the identity matrix. This allows some unused elements of the covariance matrix to be discarded, keeping the memory requirements low, the same solution used by López-Negrete and Biegler [2012, Sec. 2.2]. We also remark that the results presented in this section cannot be used to diagnose unidentifiability, as then Assum. 1 would not hold. Correlation coefficients close to \pm 1 can, however, be indicative of poorly identifiable, ill-conditioned problems, often associated with overparametrization, improper excitation, or inadequate model postulates.

\section{Application examples}

We now consider applications of the results of Sec. 2 to simulated problems in systems and control. The first example consists of the estimation of the parameter and state-path covariance in the system identification of a nonlinear continuous-time system using the collocationbased output-error method, formulated as a maximum likelihood problem. The second example is an application to joint state-path and parameter estimation in stochastic differential equations (SDEs). The estimated posterior covariance is used to calibrate a Markov chain Monte Carlo (MCMC) sampler of state-paths and parameters.

The implementation of the estimators used herein is in the open-source software package ceacoest ${ }^{1}$, the $C e n$ -

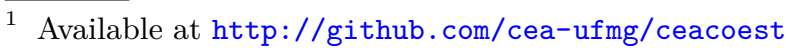


tro de Estudos Aeronáuticos Control and Estimation library, under development by the author. It has bindings for the large-scale nonlinear optimization solver IPOPT by Wächter and Biegler [2006], which was used together with the HSL Mathematical Software Library ${ }^{2}$. The code used to generate the data and analyses for this article is also available as free software ${ }^{3}$.

\subsection{Collocation-based output-error estimation}

The output-error method is a standard method for system identification and parameter estimation. Given the system parameters and initial condition, the system is simulated and the output error minimized, according to a loss function associated to a measurement noise distribution. When there is no prior distribution for the estimates it is a maximum likelihood estimator.

When implemented with collocation methods, the decision variables of the optimization problem are augmented with the state vector at all simulation points and the simulation method is enforced as as equality constraints [for a brief overview, see Dutra, 2019]. We used the trapezoidal rule for the collocation in these experiments, see Williams and Trivailo [2005] for this and many alternative methods for collocation. Betts and Huffman [2003] and Betts [2010] discuss implementation issues in detail.

To illustrate the estimation of uncertainties, we perform simulated experiments on the Van der Pol oscillator, a benchmark model for modelling nonlinear dynamics and chaos [Aguirre and Letellier, 2009, Appx. A.2]. Its dynamics is governed by the ODE

$$
\dot{x}_{1}=x_{2}, \quad \dot{x}_{2}=\mu\left(1-x_{1}^{2}\right) x_{2}-x_{1},
$$

where $x=\left[x_{1}, x_{2}\right]^{\top}$ is the state vector and $\theta=[\mu \sigma]^{\top}$ is the unknown parameter vector, to be estimated. Noisy measurements of the first state $x_{1}$ are available with variance $\sigma^{2}$. The system is simulated for the interval $t \in[0,20]$, starting from $x_{1}(0)=0$ and $x_{2}(0)=1$. The true values of the parameters, used to generate the data, are $\mu=2$ and $\sigma=0.1$. The measurements are spaced by 0.1 time units and the collocation mesh spacing is 0.01 time units.

The output error method needs an initial guess for all decision variables. The initial guess for $x_{1}$ was obtained by low-pass filtering the measurements. The guess for $x_{2}$ was the finite difference derivative of the $x_{1}$ guess. Finally, the guess of $\mu$ is obtained from the other guesses using linear least squares.

\footnotetext{
2 HSL. A collection of Fortran codes for large scale scientific computation. http://www.hsl.rl.ac.uk/

3 http://github.com/dimasad/hessinv-code
}

Table 1. Estimation results for the simulated experiment. The first column is the sample standard deviation of the estimate; the second is the mean estimated standard deviation $\hat{\Sigma}$ of each estimate, obtained from the inverse Hessians; the third is the sample standard deviation of $\hat{\Sigma}$.

\begin{tabular}{cccc}
\hline Estimate & std. dev. & mean $\hat{\Sigma}$ & std. dev. $\hat{\Sigma}$ \\
\hline$\mu$ & 0.0060 & 0.0060 & 0.0003 \\
$\sigma$ & 0.0050 & 0.0049 & 0.0003 \\
$x_{1}(0)$ & 0.0085 & 0.0085 & 0.0007 \\
$x_{2}(0)$ & 0.0463 & 0.0459 & 0.0030 \\
\hline
\end{tabular}

To evaluate the distribution of the estimates and how it relates to the uncertainty estimates obtained from the inverse Hessian, a total of 10000 different realizations of the noise were performed, one of which is shown in Fig. 1. The estimated variance $\hat{V}$ of each estimate is the corresponding element of the diagonal of $-H_{L}^{-1}$. The estimated standard deviation $\hat{\Sigma}$ is its square root. These estimated uncertainties depend on the noise, so their mean and scatter is also analyzed. The results are summarized in Table 1 for the parameter vector $\theta$ and initial state vector $x(0)$. A good agreement between the observed and estimated standard deviations was obtained. The histograms of the parameter estimates are shown in Fig. 2. By employing the approximation (17a) and the result of Lemma 6 , we can also obtain the estimate uncertainty of the whole state path, shown in Fig. 3.

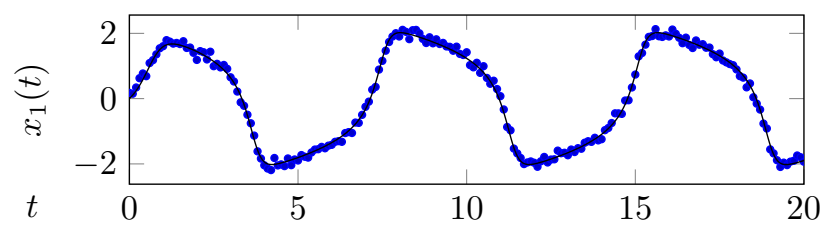

Fig. 1. Data from one realization of the Van der Pol oscillator. The true state path is the solid line and the marks are the noisy measurements.
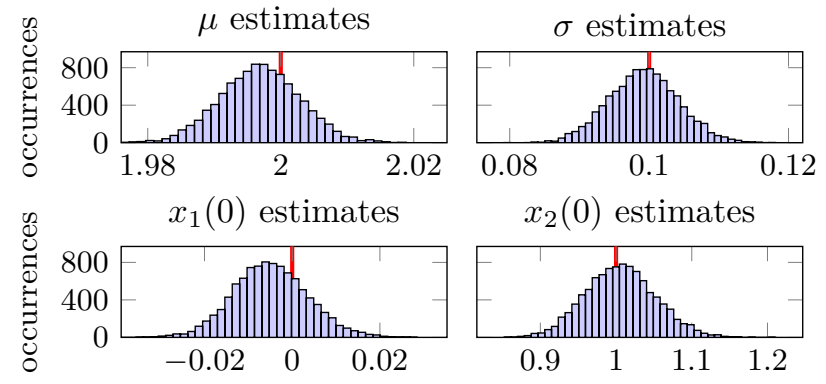

Fig. 2. Histogram of the Van der Pol parameter estimates. The red line in the background marks the true values, used to generate the data. 


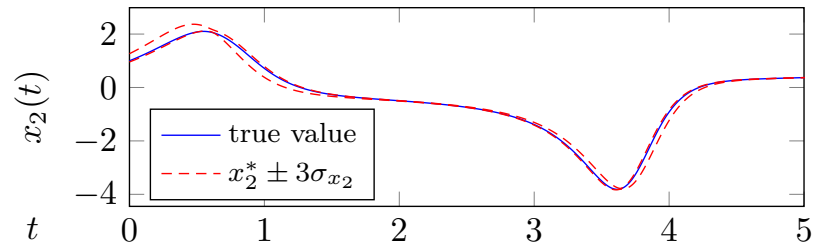

Fig. 3. Estimated $x_{2}$ path confidence bounds compared with the true simulated values for a realization of the Van der Pol experiment.

\subsection{Joint MAP state-path and parameter estimation}

For general nonlinear dynamical systems subject to noise, the posterior distribution does not assume tractable closed-form solutions. In such cases, Monte Carlo methods are a popular and powerful choice for evaluating the posterior in detail, including features such as skewness, excess kurtosis and nonlinear relationships between the variables. To that end, estimates of the posterior covariance matrix obtained from the results of Sec. 2 can aid the implementation of efficient Monte Carlo samplers. In Markov chain Monte Carlo with the random-walk Metropolis algorithm, for example, the target distribution's covariance can be used to tune the jumping scales and geometry for efficient sampling [?].

We demonstrate this application in a nonlinear continuous-time system described by a stochastic differential equation (SDE), the Duffing oscillator. It is a benchmark model for modeling nonlinear dynamics and chaos [Aguirre and Letellier, 2009] and state estimation in SDEs [Ghosh et al., 2008, Khalil et al., 2009, Namdeo and Manohar, 2007]. The example and the estimator are similar to those of a previous work [Dutra et al., 2017, Sec. 4.1]. The dynamics is described by the following stochastic differential equation

$$
\begin{aligned}
\mathrm{d} X_{t} & =\left[-A Z_{t}^{3}-B Z_{t}-D X_{t}+\gamma \cos t\right] \mathrm{d} t+\sigma_{d} \mathrm{~d} W_{t}, \\
\mathrm{~d} Z_{t} & =X_{t} \mathrm{~d} t
\end{aligned}
$$

where $X_{t}$ and $Z_{t}$ are the system states; $W_{t}$ is a Wiener process; $A, B$, and $D$ are unknown parameters, to be estimated; and $\gamma$ and $\sigma_{d}$ are known parameters.

Discrete-time measurements $Y_{k}$ of $Z_{t}$ with independent Gaussian noise were used for the estimation,

$$
Y_{k} \mid X, Z, \Theta \sim \mathcal{N}\left(Z_{k t_{s}}, \Sigma_{y}^{2}\right), \quad k=0, \ldots, N
$$

where $t_{s}$ is the sampling period, the $\mathbb{R}$-valued standard deviation $\Sigma_{y}$ is an unknown parameter, to be estimated, and $\Theta$ is the full vector of unknown parameters. Uniform priors for all parameters and initial conditions were used. The system was simulated using the strong explicit order 1.5 scheme [Kloeden and Platen, 1992, Sec. 11.2] with a time step of 0.005 . The parameters and initial states used to generate the data are shown in Table 2.
Table 2. Parameter values used to simulate the Duffing oscillator.

\begin{tabular}{cccccccccc}
\hline$X_{0}$ & $Z_{0}$ & $A$ & $B$ & $D$ & $\Sigma_{y}$ & $\gamma$ & $\sigma_{d}$ & $t_{s}$ & $T$ \\
1.0 & 1.0 & 1.0 & -1.0 & 0.2 & 0.1 & 0.3 & 0.1 & 0.1 & 200 \\
\hline
\end{tabular}

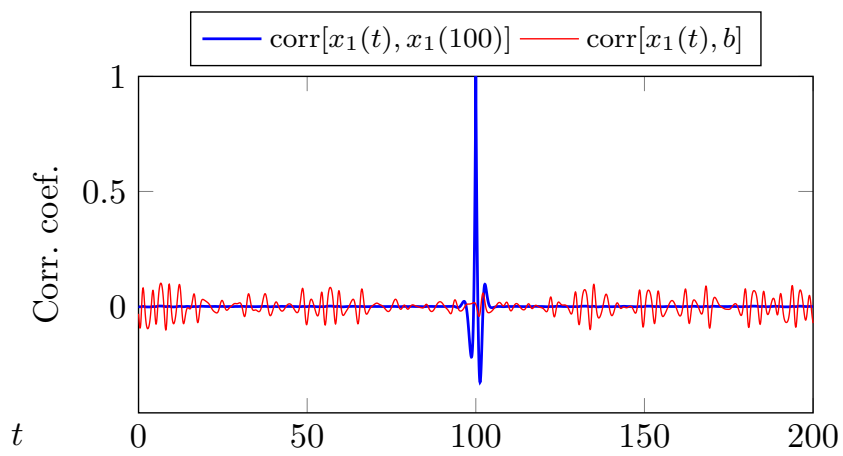

Fig. 4. Approximate posterior correlation coefficients between the state-path $x_{1}(t)$ and the $x_{1}(100)$ state and the $b$ parameter, for the Duffing oscillator.

The joint MAP state-path and parameter estimator [Dutra et al., 2017] is the solution to the following optimal control problem:

$$
\begin{aligned}
\operatorname{maximize} & \ell(x, z, \theta, \eta) \\
\text { subject to } & \dot{x}=-a z^{3}-b z-d x+\gamma \cos +\sigma_{d} \eta \\
& \dot{z}=x,
\end{aligned}
$$

with merit function

$$
\begin{aligned}
\ell(x, z, \theta, \eta)= & -\frac{1}{2} \sum_{k=0}^{N} \frac{\left[y_{k}-z\left(k t_{s}\right)\right]^{2}}{\sigma_{y}} \\
& -(N+1) \ln \sigma_{y}-\frac{1}{2} \int_{0}^{T} \eta(t)^{2} \mathrm{~d} t+\frac{T d}{2},
\end{aligned}
$$

where $x:[0, T] \rightarrow \mathbb{R}$ and $z:[0, T] \rightarrow \mathbb{R}$ are the candidate modal state-paths, $\eta$ is the associated process noise path, and $\theta=\left[\begin{array}{llll}a & b & d & \sigma_{y}\end{array}\right]^{\top}$ is the unknown parameter vector. The infinite-dimensional problem (18) needs to be discretized into finite-dimensional NLP for solution. To do that, we used the same collocation method used to implement the output-error in Sec. 3.1, the trapezoidal method. For some alternatives discretizations and implementation details, see Betts [2010].

From the approximate covariance matrix, obtained from $H_{L}^{-1}$, we can inspect the posterior correlation coefficients, shown in Fig. 4. These show important aspects of the problem: the states are highly correlated with their neighbours, across a time window; and the parameters are correlated with the states across all time instants. These dependencies must be taken into account in the Metropolis-Hastings algorithm for good acceptance rates to be obtained [Robert and Casella, 2004, p. 327].

A random perturbation of the modal estimate was used to initialize a hybrid Gibbs sampler [Robert and 
Casella, 2004, Sec. 10.3] for the posterior distribution of the state-path and parameters. We used Gibbs sampling to explore the local features of the posterior with a full random-walk Metropolis step after each 15 Gibbs cycles to increase diversity and better explore the global support of the distribution. Since the conditional distribution of each variable does not admit a tractable form, Metropolis-within-Gibbs [Robert and Casella, 2004, Sec. 10.3.3] was used to sample the Gibbs steps.

In this problem, $\theta, z(0)$ and $x\left(t_{0}\right), \ldots, x\left(t_{N}\right)$ are a possible choice for the independent variables $p \in \mathbb{R}^{n}$. For the $i$-th independent variable, given its value at the $j$-th step of the chain $p_{i}^{(j)}$, the Metropolis-within-Gibbs candidate was generated as

$$
\tilde{p}_{i}^{(j+1)}=p_{i}^{(j)}+3.2 \epsilon_{i j}, \quad \epsilon_{i j} \sim \mathcal{N}\left(0, R_{i i}\right),
$$

where the approximate covariance $R:=-\left(\nabla^{2} \tilde{\ell}\right)^{-1}$ is the inverse reduced Hessian, obtained from Thm. 3. For the full Metropolis step, the candidate was generated according to

$$
\tilde{p}^{(j+1)}=p^{(j)}+\frac{1.8}{\sqrt{n}} \epsilon_{j}, \quad \epsilon_{j} \sim \mathcal{N}(0, R) .
$$

These candidates were then accepted or rejected with the Metropolis algorithm acceptance probability. The scale factors were tuned starting with the values recommended by ? and yielded an average acceptance rate of $26.8 \%$, close to the $23.4 \%$ suggested by Roberts et al. [1997].

\section{Conclusions}

In this article, we showed how to approximate estimate uncertainties in equality-constrained MAP and maximum likelihood estimation. These estimators have various applications in system identification and state estimation, and methods which allow uncertainty estimation directly from the augmented problem can help in their adoption by a wider userbase. Two example applications of the results in systems and control were presented, covering both MAP and ML estimation.

\section{References}

L.A. Aguirre and C. Letellier. Modeling nonlinear dynamics and chaos: a review. Math. Probl. Eng., 2009: 238960, 2009.

T. Ando. Bayesian model selection and statistical modeling. Chapman and Hall/CRC, 2010.

C. Andrieu, A. Doucet, S.S. Singh, and V.B. Tadić. Particle methods for change detection, system identification, and control. Proc. IEEE, 92(3):423-438, 2004.

Y. Bard. Nonlinear parameter estimation. Academic Press, 1974.
D.S. Bernstein. Matrix Mathematics: Theory, Facts, and Formulas. Princeton University Press, 2nd edition, 2009.

J.T. Betts. Practical methods for optimal control and estimation using nonlinear programming. SIAM, 2nd edition, 2010.

J.T. Betts and W.P. Huffman. Large Scale Parameter Estimation Using Sparse Nonlinear Programming Methods. SIAM J. Optim., 14(1):223-244, 2003.

H.G. Bock. Numerical Treatment of Inverse Problems in Chemical Reaction Kinetics. In Modelling of Chemical Reaction Systems: Proceedings of an International Workshop, pages 102-125, 1980.

H.G. Bock. Recent Advances in Parameteridentification Techniques for O.D.E. In Numerical Treatment of Inverse Problems in Differential and Integral Equations: Proceedings of an International Workshop, pages 95121, 1983.

J.J. Boisvert, M.W. Donaldson, and R.J. Spiteri. Solving Parameter Estimation Problems with SOCX. In Control and Optimization with Differential-Algebraic Constraints, chapter 12, pages 253-272. SIAM, 2012.

P.E. Caines and L. Ljung. Prediction error estimators: Asymptotic normality and accuracy. In Proceedings of the 1976 Joint Automatic Control Conference, 1976.

J.V Candy. Bootstrap particle filtering. IEEE Signal Proc. Mag., 24(4):73-85, 2007.

H. Cramér. Mathematical Methods of Statistics. Princeton University Press, 1946.

D.A. Dutra, B.O.S. Teixeira, and L.A. Aguirre. Joint maximum a posteriori smoother for state and parameter estimation in nonlinear dynamical systems. In SYSID, pages 900-905, 2012.

D.A. Dutra, B.O.S. Teixeira, and L.A. Aguirre. Maximum a posteriori state path estimation: Discretization limits and their interpretation. Automatica, 50 (5):1360-1368, 2014.

D.A.A. Dutra. Collocation-Based Output-Error Method for Aircraft System Identification. In Proc. AIAA Aviation 2019 Forum, page 3087, 2019.

D.A.A. Dutra, B.O.S. Teixeira, and L.A. Aguirre. Joint maximum a posteriori state path and parameter estimation in stochastic differential equations. Automatica, pages 403-408, 2017.

A. Gelman, G.O. Roberts, and W.R. Gilks. Efficient Metropolis jumping rules. In J. M. Bernardo, J.O. Berger, A.P. Dawid, and A.F.M. Smith, editors, Bayesian Statistics 5, pages 599-607. Oxford University Press, 1996.

S. J. Ghosh, C. S. Manohar, and D. Roy. A sequential importance sampling filter with a new proposal distribution for state and parameter estimation of nonlinear dynamical systems. Proc. R. Soc. A, 464(2089): 25-47, 2008.

R.V. Jategaonkar. Flight Vehicle System Identification. AIAA, 2nd edition, 2015.

R. Kashyap. Maximum likelihood identification of stochastic linear systems. IEEE Trans. Autom. Control, 15(1):25-34, 1970. 
M. Khalil, A. Sarkar, and S. Adhikari. Nonlinear filters for chaotic oscillatory systems. Nonlinear Dynam., 55 (1-2):113-137, 2009.

M. Klaas, M. Briers, N. de Freitas, A. Doucet, S. Maskell, and D. Lang. Fast particle smoothing: If I had a million particles. In ICML 2006, pages 25-29, 2006.

V. Klein and E.A. Morelli. Aircraft system identification. AIAA, 2006.

P.E. Kloeden and E. Platen. Numerical Solution of Stochastic Differential Equations. Springer, 1992.

S.G. Krantz and H.R. Parks. The Implicit Function Theorem: History, Theory, and Applications. Birkhäuser Basel, 2003.

L. Ljung. System Identification: Theory for the User. Prentice Hall, 2nd edition, 1999.

R. López-Negrete and L.T. Biegler. A Moving Horizon Estimator for processes with multi-rate measurements: A Nonlinear Programming sensitivity approach. J. Process Contr., 22(4):677-688, 2012.

R.E. Maine and K.W. Iliff. The Theory and Practice of Estimating the Accuracy of Dynamic FlightDetermined Coefficients. NASA Reference Publication 1077, 1981.

R. Mehra. Optimal input signals for parameter estimation in dynamic systems - Survey and new results. IEEE Trans. Autom. Control, 19(6):753-768, 1974.

P.C. Murphy. An Algorithm for Efficient Maximum Likelihood Estimation and Confidence Interval Determination in Nonlinear Estimation Problems. PhD thesis, George Washington University, 1985.

V. Namdeo and C.S. Manohar. Nonlinear structural dynamical system identification using adaptive particle filters. J. Sound and Vib., 306(3-5):524-563, 2007.

J. Nocedal and S.J. Wright. Numerical Optimization. Springer-Verlag New York, 2006.

H. Pirnay, R. López-Negrete, and L.T. Biegler. Optimal sensitivity based on ipopt. Math. Program. Comput., 4(4):307-331, 2012.

J.O. Ramsay, G. Hooker, D. Campbell, and J. Cao. Parameter estimation for differential equations: a generalized smoothing approach. J. R. Stat. Soc. B, 69(5): 741-796, 2007.

A.H. Ribeiro and L.A. Aguirre. Shooting methods for parameter estimation of output error models. In Proc. 20th IFAC World Congress, pages 13998-14003, 2017.

A.H. Ribeiro, K. Tiels, J. Umenberger, T.B. Schön, and L.A. Aguirre. On the smoothness of nonlinear system identification. Automatica, 2019. Provisionally accepted, arXiv: 1905.00820.

C. Robert and G. Casella. Introducing Monte Carlo Methods with R. Springer, 2010.

C.P. Robert. The Metropolis-Hastings Algorithm. Wiley, 2015.

C.P. Robert and G. Casella. Monte Carlo Statistical Methods. Springer, 2 edition, 2004.

G.O. Roberts, A. Gelman, and W.R. Gilks. Weak convergence and optimal scaling of random walk Metropolis algorithms. The Annals of Applied Probability, 7(1):110-120, 1997.
P. Stoica and T. Söderström. On non-singular information matrices and local identifiability. Int. J. Control, 36(2):323-329, 1982.

T. Söderström. Comments on "Order assumption and singularity of information matrix for pulse transfer function models". IEEE Trans. Autom. Control, 20 (3):445-447, 1975.

A.W. van der Vaart. Asymptotic Statistics, volume 3. Cambridge University Press, 1998.

A. Wächter and L.T. Biegler. On the implementation of an interior-point filter line-search algorithm for largescale nonlinear programming. Math. Program., 106 (1):25-57, 2006.

A. Wald. Note on the Consistency of the Maximum Likelihood Estimate. Ann. Math. Statist., 20(4):595601, 1949.

S.S. Wilks. Mathematical Statistics. John Wiley \& Sons, 1962.

P. Williams and P. Trivailo. Optimal parameter estimation of dynamical systems using direct transcription methods. Inverse Problems in Science and Engineering, 13(4):377-409, 2005. 